\title{
Bistable properties of a memory-related gene regulatory network
}

\author{
Gonzalo S. Nido \\ Department of Computer Science \\ University of Otago, New Zealand \\ Email: gsnido@cs.otago.ac.nz
}

\author{
Joanna M. Williams \\ Department of Anatomy and Structural Biology \\ Brain Health and Repair Research Centre \\ University of Otago, New Zealand \\ Email: joanna.williams@stonebow.otago.ac.nz
}

\author{
Lubica Benuskova \\ IEEE Member \\ Department of Computer Science \\ University of Otago, New Zealand \\ Email: lubica@cs.otago.ac.nz
}

\begin{abstract}
Long-term potentiation (LTP) is a long-lasting enhancement in signal transmission between two neurons, and represents a widely accepted experimental model for long-term memory processes. Although it is now clear that the maintenance of LTP requires new gene transcription, little is known on the genetic mechanisms underlying these changes. We assume that an LTP-related gene regulatory network has two equilibrium states in terms of gene expression levels which correspond to a pre- and post-LTP states. This network is shifted from the first to the latter by means of a perturbation, which experimentally corresponds to the high-frequency stimulus necessary to induce LTP in vivo. Based on this assumption and by means of modeling the transcriptional regulation with weight matrices, we study the properties of the main LTP-related network recently proposed in [1]. First, we classify the LTP-related genes according to their relevance to the bistable dynamic output of the network. In addition, we demonstrate how the LTP gene regulatory network architecture holds a higher tendency towards bistable behaviours than we should expect of a random network.

Index Terms-Biology and genetics, Computational neuroscience, Systems theory.
\end{abstract}

\section{INTRODUCTION}

Memory processes in the brain are dependent on the capacity of neurons to undergo long-lasting enhancement of synaptic effectiveness. Long-term potentiation (LTP) is one of such mechanisms, providing a cellular model of associative, specific, and long-lasting storage.

Although LTP is generally divided into three phases over time only the intermediate- and late- phases (often referred as LTP2 and LTP3 respectively), seem to be dependent on protein synthesis. As well as long-term memory, the late-phase LTP is dependent on both changes in gene expression and protein synthesis [2]-[5].

The new transcripts and proteins that characterise the lateLTP could be targeted to the specific synapses from the nucleus by means of a synaptic "tag" generated at the potentiated synapses [6]. A first wave of transcription brought about by the activated constitutive transcription factors (TFs) would consist of effector genes and inducible TFs which, in turn, would be responsible for a second wave of transcription [1], [3].

Although several genes have been identified to belong to some of those categories (see [3], [4], [7] for some examples) it is unclear how do they coordinate in order to lead to the persistence of LTP. Several efforts have been taken towards defining a wider picture of the biochemical networks underlying the maintenance of LTP [1], [8]-[11]. The pathways by which the genomic response is elicited seem to be triggered by the activation by phosphorylation of cAMP response element-binding protein (CREB) by the phosphorylated calcium-dependent and cAMP-dependent kinase signaling pathways. CREB funtions as a hub in the gene regulation. Its phosphorylation can be driven by a plethora of different kinases [12] and in turn, its activation leads to the expression of transcriptionally linked genes.

Once phosphorylated, CREB acts as a TF stimulating the expression of other TFs and other effector genes by binding to the promoter CRE region. The transciptional response will constitute a first wave of gene expression. Other TFs which are constitutively expressed may also become active following LTP induction (Elk-1 and Srf are among the candidates).

The first wave of de novo transcription brought about by the LTP-activated TFs consists of activity-induced TFs, structural proteins, signal transduction proteins, growth factors, and enzymes. This set of genes has been referred to as immediateearly genes (IEGs). Some of the induced TFs among the IEGs are c-FOS, JUN, EGR1,2,3, genes from the AP1 family and LIRF, which are believed to drive the expression of delayed effector genes. Many other IEGs have been reported as "effector IEGs"- ARC, HOMER 1a, tPA, NARP, BDNF, Arcadlin, and Rheb are just some examples (see [3], [4], [13], [14] for comprehensive reviews). While different putative functions have been proposed for these effector genes (gene expression regulation, cellular growth and adhesion, cell cycle...), it seems clear that they are part of the coordinated process of LTP consolidation.

Several studies have more recently covered the gene expression activation with studies of corregulation based on microarrays (see for example [1], [9]), identifying sets of differentially expressed genes after the induction of LTP. It appears that the expression state of the LTP-related transcriptional regulatory network (LTP-GRN) shifts from a resting state (pre-LTP state) to a post-LTP state, where LTP has been induced via experimental high frequency stimulus (HFS). These expression states have been measured experimentally using high density arrays by Ryan et al. [1]. By using Ingenuity pathway analysis (IPA) [15] with the set of differentially 


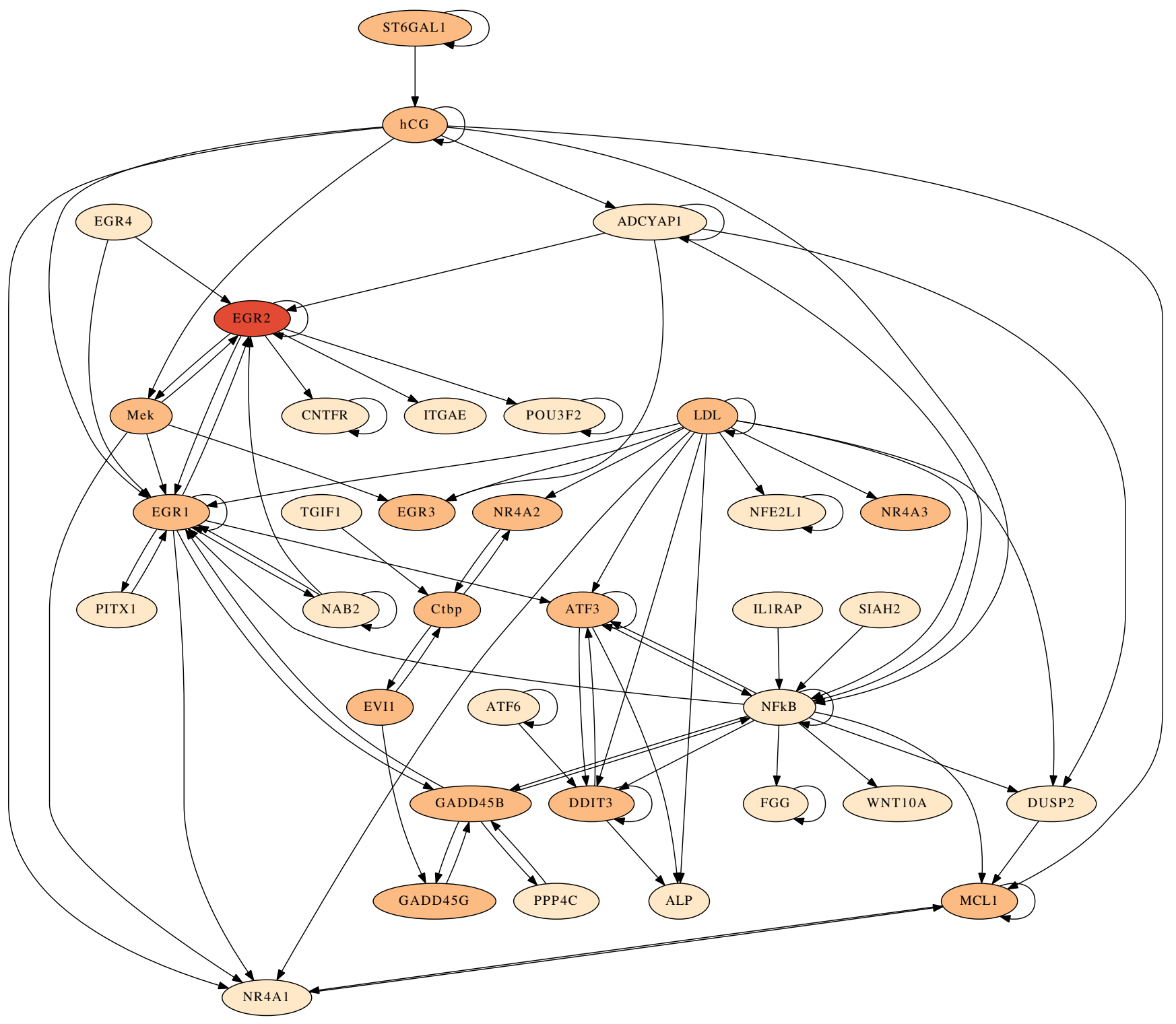

Fig. 1. LTP-GRN as reported in [1]. The colors in the nodes represent the knock-out effects on the bistable output frequency of the LTP-GRN - the shade of red, indicates how the knock-out of that gene affects the ability of the network to reach bistable outputs $\left(P_{B}\right)$. Darker nodes are therefore of a crucial importance for the network to show an expression landscape compatible with the two-attractors hypothesis. The EGR family seems to be of a fundamental importance for the bistable properties of the LTP-GRN. (Red nodes, $0.01<P_{B} \leq 0.03$, Orange nodes, $0.03<P_{B} \leq 0.05$, Light orange nodes, $0.05<P_{B}<0.08$ ).

expressed genes, they identified biologically relevant networks related with LTP. The IPA software queries a manually curated database of functions and interactions obtained from scientific literature.

Among the three highest scoring networks, their results suggest that some genes may play central roles during LTP induction without showing a significant alteration in their expression levels (such is the case of NF-kB). Other genes identified as major hubs in their LTP-regulated gene expression networks were SRF, EGR1 and CREB. ERK also appears as a central hub in the network and its activity seems regulated by the phosphatase activity of DUSPs, which in turn appear to have a coordinated upregulation. In addition, DUSP and
EGR families regulate the MAPK signaling pathway, which is known to have an essential role in LTP.

In the present work, we analyse the most significant network published in [1] to investigate its topological properties from a dynamical perspective. We model the transcription regulation through weight matrices as described by Weaver et al [16], a methodology which can be regarded as a discrete approximation of a Hopfield model with graded response (see Section II-A). We show that the particular topology of the LTP-GRN is more likely to hold bistability when compared to random networks. On a similar perspective, we classify the LTP-related genes according to their relevance to the bistable output of the network. 


\section{MAterials AND MEthods}

\section{A. Dynamics with weight matrices}

Continuous models of GRNs based on differential equations entail a main disadvantage compared to discrete-time approximations. Despite experimental evidence can be gathered regarding the networks' topology, there is a lack of quantitative information in relation to the biochemical parameters underlying gene interactions. In other words, the strength of the interactions between genes and proteins are difficult to measure. On the other hand, the widely-used Boolean models for gene regulation might oversimplify the complexity underlying biological interactions.

For this study, we have adopted the TReMM algorithm (Transcription Regulation Modeled with Matrices) described by Weaver et al. [16]. The TReMM methodology characterises the gene expression values from a state of maximal expression to a state of minimal repression. These intervals are genespecific, and during a simulation a gene can take any continuous value in the range defined by its maximal and minimal expression.

The gene expression state of a network is represented by a vector $u(t)$, where $u_{i}$ corresponds to the expression level of the $i$ th gene. The regulatory interactions between genes are modeled with a weight matrix, $W$ so that the effect of gene $j$ on gene $i$ is the expression level of $j\left(u_{j}\right)$ times its regulatory influence on $i, w_{i, j}$. This regulatory influence can be either activating $\left(w_{i, j}>0\right)$, or repressing $\left(w_{i, j}<0\right)$. The values of $w_{i, j}=0$ correspond to the lack of experimental evidence for the effect of gene $j$ on gene $i$. Put differently, only the nodes connected in Fig. I have nonzero values of $W$.

The total regulatory input to $i, r_{i}(t)$, is calculated by adding all the genes which interact with it:

$$
r_{i}(t)=\sum_{j} w_{i, j} u_{j}(t)
$$

The expression $u_{i}(t+1)$ of a gene $i$ to the regulatory input $r_{i}(t)$ is "squashed" with a sigmoidal function,

$$
u_{i}(t+1)=\frac{m_{i}}{1+e^{-\left(\alpha_{i} r_{i}(t)+\beta_{i}\right)}}
$$

where $m_{i}$ corresponds to the maximal observed expression level for the gene $i$, and the parameters $\alpha_{i} \in \mathbb{R}_{\leq 0}, \beta_{i} \in \mathbb{R}$, and $w_{i, j} \in \mathbb{R}$ correspond to the $i$ th's gene's intrinsic response to the regulatory inputs (or slope of the sigmoidal response function), the gene's basal expression level, and the relative weights of the interactions respectively. Note that the $\alpha_{i}$ constant can only take positive values. The expression levels of all $N$ genes $u_{i}(t)$ are updated simultaneously in the time course experiments. The simulations converge to a stable state of unchanging gene expression or cyclical set of gene expression states. We show in Fig. 2 a only a subset of 11 genes of the whole network for the sake of clarity.

This methodology can be regarded as a modification of a recurrent Hopfield network with graded response and synchronous updates. The mathematical condition for the weight

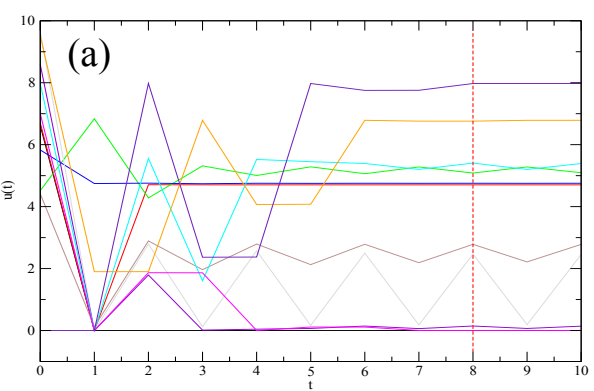

(b)

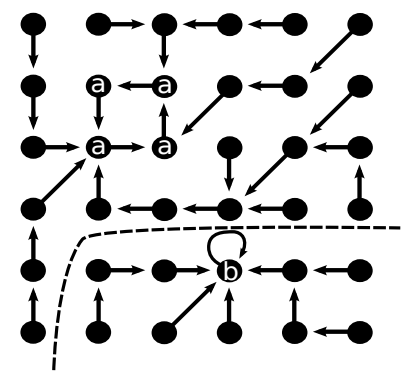

Fig. 2. (a) Example dynamics of the LTP-GRN reaching a steady state at $t=8$. Only a subset of 11 genes from the total 35 genes is shown for the sake of clarity. The vertical axis represents the level of gene expression $u(t)$, the horizontal axis represents iterations. (b) Schematic representation of a small region in the gene expression states space. Each node represents a $u(t)$ vector, and the edges connect a state in time $t$ with the state in time $t+1$. Owing to the deterministic nature of the dynamic modeling used in the study, if a state $u(t)$ is visited at any point during the simulation, only one transition is possible for $u(t+1)$. In the scheme, two hypothetic attractors are depicted (nodes marked with a and b), delimiting two basins of attraction (dashed line). They represent a cyclic and a fixed point attractor respectively.

matrix imposed in the original Hopfield model (symmetric $W$ with zero diagonal) [17] is eliminated, allowing self-loops and asymmetric interactions.

The convergence to a particular stable state is dependent on the initial conditions $u(t=0)$, and on the gene expression landscape described by the parameters $\alpha, \beta$, and $W$ for the network studied. These landscapes are constituted by basins of attraction, a set of states that are attracted to a fixed point, and different set of parameters produce different basins of attraction. We are interested in the existence of a particular landscape for the LTP-GRN, where the basins of attraction would represent the pre- and post-LTP cell states, potentially accessible from different initial conditions as represented in Fig. 2b. Those initial conditions could be specified by the cell's environment (such as the high frequency stimulation).

This view of a gene regulatory network is analogous to a content-adressable memory (CAM), which restores the preand post-LTP patterns by supplying some subpart of the memory in the inherently noisy environment of the cell.

For a given network (either the LTP-GRN or a random topology) the parameter space for $\alpha, \beta$, and $W$ is evenly explored by 10,000 random tries. This procedure characterises the network resulting in an average frequency of bistable outputs $\left(P_{B}\right.$, see section II-C) which should be free of the bias 
introduced by the choice of a particular set of parameters.

The TReMM methodology have the advantage of representing gene regulation with a considerable degree of complexity while still being computationally tractable for the size of the network studied.

\section{B. LTP-GRN and gene expression data}

For this study we have chosen the highest scoring LTP-GRN published in [1], constituted by a total of 35 genes (see Fig. I), which was constructed using Ingenuity Pathway network analysis tools [15] and is based on biological information sourced from literature references.

The initial conditions for the time course experiments, $u(t=0)$, as well as the maximal expression levels $m$ were obtained from real expression data - the values for the postLTP state correspond to the microarray hybridized with tissue where LTP was induced by high-frequency simulus (HFS), whereas the pre-LTP state expression was obtained from the control microarrays (no HFS).

\section{Frequency of bistable outputs $P_{B}$}

We define as bistable a network dynamics which converges onto two different asymptotic states when initialised with two different sets of expression values. In this study, we have set those starting expression values according to the LTPinduced microarray data and the control microarray data, namely, running for each network and each set of $\alpha, \beta$, and $W$ parameters two parallel dynamics representing pre- and post-LTP conditions respectively, as summarized in Fig. 2b. If each of the two sets of initial conditions fall in different basins of attraction, the dynamics will lead to different terminal states. This cases are referred to as bistable outputs $(B)$. We have chosen the experimental expression values obtained by Ryan et al. [1] since they represent real estimates of the gene expression levels.

By sampling the space of parameters and testing whether the output states of the pre- and post-LTP parallel dynamics reach different points in the $\mathrm{N}$-dimensional space, we calculate the frequency of bistable outputs or $P_{B}$, which characterises the network's expression state space around the pre- and post-LTP initial conditions. In this work, we have randomly sampled the space of parameters by 10,000 trials with $w_{i, j} \in[-1,+1]$, $\beta_{i} \in[-1,+1]$, and $\alpha \in(0,+3]$.

The value of $P_{B}$ is free of the bias which a particular set of parameters would introduce, and serves as a descriptor of how a particular topology is likely to show a sensitivity to the realistic initial conditions observed in the in vivo experiments. Put differently, the attractors in the gene expresion states space represent stable phenotypes, while $W$ represents the neuron's genotype.

\section{Random and knock-out networks}

In order to characterise the LTP-GRN, we have constructed a total of 100 random networks by preserving the same number of nodes and edges as in the LTP-GRN and without assuming any particular degree distribution. These random

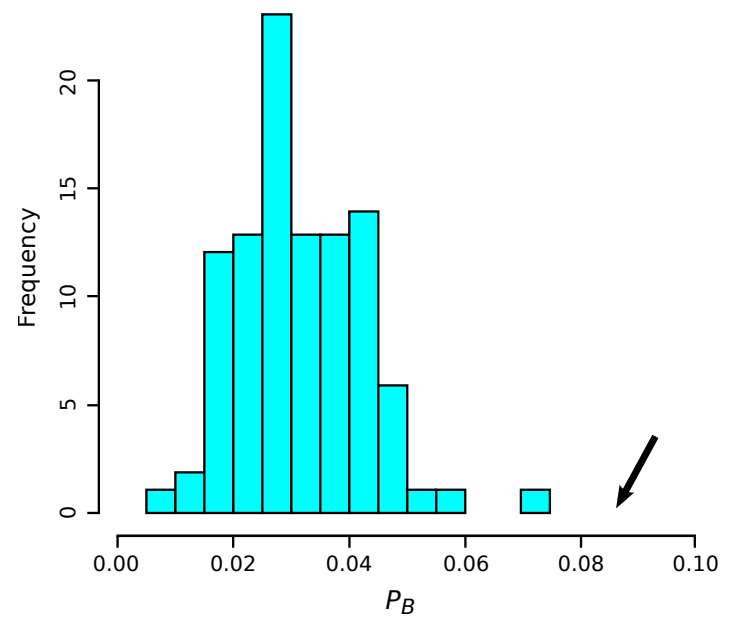

Fig. 3. Distribution of 100 random networks according to their bistable output frequencies $P_{B}$ (10,000 simulations for each network in the parameter space). The $P_{B}$ distribution for the random networks has $\mu=0.031$ and $\sigma=0.011$. The LTP-related network shows a much higer $P_{B}=0.086$, which lies about 5 standard deviations away from the mean of the random networks' distribution (black arrow).

networks have been characterised in terms of their $P_{B}$, and the values are represented in Fig. 3. Then we run a set of 10,000 simulations with the LTP-GRN with the fixed topology shown in Fig. I, with random values of weight coefficients $W, \alpha$, and $\beta$ and we calculated $P_{B}$.

Similarly, we have calculated the $P_{B}$ for the 35 networks resulting from the knock-out of each gene in the LTP-GRN. Some of these knock-out networks show an inferior propensity to bistability (a lower $P_{B}$ ), meaning that the removed gene has a key role in the capacity of the LTP-GRN to reach bistable outputs. The $P_{B}$ values for the removal of each gene are represented in Fig. I, where a darker shade of red means higher influence in the network's bistability propensity.

\section{RESULTS}

\section{A. LTP-GRN bistable frequency}

The frequency of bistable outputs for the LTP-GRN $\left(P_{B}=\right.$ 0.086 ) is in sharp contrast with the frequencies obtained for the random networks with the same number of nodes and edges (Fig. 3, avg. $P_{B}=0.031$ ). Furthermore, the value of the $P_{B}$ for the LTP-GRN is higher than any of the random networks. Assuming normality for the distribution of $P_{B}$ in the random networks' population, $(p-$ value $=0.019$, Shapiro-Wilk test $)$, the probabilty of a random network with a higher $P_{B}$ than the LTP-GRN is extremely low $\left(P<3.65 \times 10^{-07}\right)$.

\section{B. Knock-out effects}

For each $i$ th gene of the $N=35$ genes in the LTP-GRN, an independent set of 10,000 knock-out runs were performed. In these runs, the $i$ th gene of the network was removed and not considered in the simulations, setting the $i$ th row and column of $W$ to zero. The resulting 35 networks with $N=34$ were characterized in terms of their frequency of bistable outputs, $P_{B}$. 
Fig. I summarises these results by showing in a red scale the effect of removing that particular gene from the network. NF$\mathrm{kB}$ has a low influence on $\mathrm{PB}$, while the EGR members appear to be of a fundamental importance, which supports previous findings [18]. Interestingly, the level of expression of NF-kB does not change after LTP induction [1].

\section{DISCUSSION}

Evolution has equipped cells with a robust and plastic analog system which confers to living cells the ability to respond in a graded manner to the environmental inputs. However, some aspects of the cellular behaviour are of a switch-like nature - this is the case of cell cycle checkpoints, cell-fate transitions, and apoptosis to name a few. Likewise, LTP as a long-lasting phenotypic phenomenon represents one of such Boolean characteristics, and its maintenance relies on gene expression.

Back in 1969, Stuart Kauffman proposed that cell types are attractors in the gene expression state space [19]. Similarly, our hypothesis extends this idea to the phenotypic pre- and post-LTP neuronal states, complementing other genetic and epigenetic effectors. The transition from one attractor to the other would be driven by the HFS.

From such a perspective, we classify in this study the LTP-related genes according to their relevance to the bistable dynamic output of the network. It is noteworthy how the EGR family stands out in Fig. I. Their role in LTP has been already documented [18], [20], [21], and the results presented here only stress their importance in the dynamic context of the other genes. In particular, EGR2 appears to be the most determinant gene for the network to show bistability. Note however that this ranking only represents the relative importance of the genes for the bistable behaviour. For example, NF-kB shows a relatively low influence on the network's $P_{B}$, while it is believed to be involved actively in LTP and synaptic plasticity [22], [23]. The low $P_{B}$ shown by NFkB is not trivial, since it also constitutes a hub in the network, and a high dependance on it for the bistable frequency might be expected. Interestingly, NF-kB does not show an altered expression level after LTP induction [1].

Since biochemical systems are impossible to be fully described due to their inherent complexity and the lack of detailed biochemical data, simplified representations are needed to approach their study. In the present work the coarse representation of the biochemical network is constituted by genes and their causal relationships retrieved automatically from databases of experimental evidences. Despite the limitations of network reconstruction and modeling [24], GRNs have the advantage of being constituted by genes, functional entities which can be experimentally targeted. Predictions from the in silico gene knock-out could be eventually tested in vivo.

In conclusion, the capacity of showing different stable points in the gene expression state space must be of a crucial importance for the GRNs involved in such step-like responses. We show that this is the case for an LTP-related GRN by using realistic expression values from microarray data.
Whether this property of the LTP-GRN extends to most biological networks or only to the networks involved in Boolean decisions remains unclear and deserves further insight. Indeed, a long-lasting cellular process such as LTP is expected to function under a changing environment. Neurons are subject to both internal and environmental noise, which compromises the stability of the gene expression states. The role of gene expression attractors in robustness and evolvability of biological networks, from a more general perspective, emerges as an interesting generalisation of this analysis, but beyond the scope of this study and will be addressed in future work.

\section{ACKNOWLEDGMENT}

This reseach was funded by a University of Otago Phd scholarship to GSN.

\section{REFERENCES}

[1] M. Ryan, S. Mason-Parker, W. Tate, W. Abraham, and J. Williams, "Rapidly induced gene networks following induction of long-term potentiation at perforant path synapses in vivo," Hippocampus, vol. 21, no. 5, pp. 541-553, 2011 .

[2] P. Nguyen, T. Abel, and E. Kandel, "Requirement of a critical period of transcription for induction of a late phase of ltp," Science, vol. 265, no. 5175 , p. $1104,1994$.

[3] W. Abraham and J. Williams, "Properties and mechanisms of ltp maintenance," The Neuroscientist, vol. 9, no. 6, p. 463, 2003.

[4] _ "Ltp maintenance and its protein synthesis-dependence," Neurobiology of Learning and Memory, vol. 89, no. 3, pp. 260-268, 2008.

[5] C. Alberini, "Transcription factors in long-term memory and synaptic plasticity," Physiological Reviews, vol. 89, no. 1, p. 121, 2009.

[6] U. Frey and R. Morris, "Synaptic tagging and long-term potentiation," Nature, vol. 385, no. 6616, pp. 533-536, 1997.

[7] M. Lynch, "Long-term potentiation and memory," Physiological Reviews, vol. 84, no. 1, p. 87, 2004.

[8] P. Lee, J. Cohen, K. Becker, and R. Fields, "Gene expression in the conversion of early-phase to late-phase long-term potentiation," Annals of the New York Academy of Sciences, vol. 1048, no. 1, pp. 259-271, 2005.

[9] C. Park, R. Gong, J. Stuart, and S. Tang, "Molecular network and chromosomal clustering of genes involved in synaptic plasticity in the hippocampus," Journal of Biological Chemistry, vol. 281, no. 40, p. 30195, 2006.

[10] B. Håvik, H. Røkke, G. Dagyte, A. Stavrum, C. Bramham, and V. Steen, "Synaptic activity-induced global gene expression patterns in the dentate gyrus of adult behaving rats: Induction of immunity-linked genes," Neuroscience, vol. 148, no. 4, pp. 925-936, 2007.

[11] L. Valor and A. Barco, "Hippocampal gene profiling: Toward a systems biology of the hippocampus," Hippocampus, 2010.

[12] S. Schulz, H. Siemer, M. Krug, and V. H

"ollt, "Direct evidence for biphasic camp responsive element-binding protein phosphorylation during long-term potentiation in the rat dentate gyrus in vivo," The Journal of Neuroscience, vol. 19, no. 13, p. 5683, 1999.

[13] T. Miyashita, S. Kubik, G. Lewandowski, and J. Guzowski, "Networks of neurons, networks of genes: an integrated view of memory consolidation," Neurobiology of Learning and Memory, vol. 89, no. 3, pp. 269-284, 2008.

[14] W. Tischmeyer and R. Grimm, "Activation of immediate early genes and memory formation," Cellular and Molecular Life Sciences, vol. 55, no. 4, pp. 564-574, 1999.

[15] I. Systems. (2011) Ingenuity pathway analysis, version 7. [Online]. Available: www.ingenuity.com

[16] D. Weaver, C. Workman, and G. Stormo, "Modeling regulatory networks with weight matrices," in Pacific symposium on biocomputing, vol. 4 Citeseer, 1999, pp. 112-123.

[17] J. Hopfield, "Neurons with graded response have collective computational properties like those of two-state neurons," Proceedings of the National Academy of Sciences, vol. 81, no. 10, p. 3088, 1984. 
[18] W. Abraham, S. Mason, J. Demmer, J. Williams, C. Richardson, W. Tate, P. Lawlor, and M. Dragunow, "Correlations between immediate early gene induction and the persistence of long-term potentiation," Neuroscience, vol. 56, no. 3, pp. 717-27, 1993.

[19] S. Kauffman, "Homeostasis and differentiation in random genetic control networks," Nature, vol. 224, pp. 177-178, 1969.

[20] J. Williams, M. Dragunow, P. Lawlor, S. Mason, W. Abraham, J. Leah, R. Bravo, J. Demmer, and W. Tate, "Krox20 may play a key role in the stabilization of long-term potentiation," Molecular Brain Research, vol. 28 , no. 1 , pp. 87-93, 1995.
[21] M. Jones, M. Errington, P. French, A. Fine, T. Bliss, S. Garel, P. Charnay, B. Bozon, S. Laroche, and S. Davis, "A requirement for the immediate early gene zif268 in the expression of late ltp and long-term memories,' Nature Neuroscience, vol. 4, no. 3, pp. 289-296, 2001.

[22] R. Freudenthal, A. Romano, and A. Routtenberg, "Transcription factor nf- $\kappa$ b activation after in vivo perforant path ltp in mouse hippocampus," Hippocampus, vol. 14, no. 6, pp. 677-683, 2004.

[23] A. O'Mahony, J. Raber, M. Montano, E. Foehr, V. Han, S. Lu, H. Kwon, A. LeFevour, S. Chakraborty-Sett, and W. Greene, "Nf- $\kappa$ b/rel regulates inhibitory and excitatory neuronal function and synaptic plasticity," Molecular and Cellular Biology, vol. 26, no. 19, pp. 7283-7298, 2006.

[24] J. Goncalves and S. Warnick, "System-theoretic approaches to network reconstruction," Control Theory and Systems Biology, p. 265, 2010. 